\title{
Matrix Blank Description as Part of Module for Machining Parameters Generation along the Tool Path for Peripheral Milling
}

\author{
Slobodan Ivanović ${ }^{*}$, Aleksandra Petrović ${ }^{2}$, Ljubomir Lukić ${ }^{2}$ \\ ${ }^{1}$ High technical mechanical school Trstenik (Republic of Serbia) \\ ${ }^{21}$ Faculty of Mechanical and Civil Engineering in Kraljevo, University of Kragujevac, Kraljevo (Republic of Serbia)
}

Peripheral pocket or contour milling using flat end milling tool can be performed using different tool paths. Engagement angle, radial and axial depth of cut are key machining parameters which influence cutting force value and directly depend of tool path itself. Knowledge of these parameters values along the tool path is necessary for simulation and monitoring of cutting force during machining process. For that purpose, module for machining parameters generation along tool path is developed and briefly explained here.

Key machining parameters are generated based on workpiece - tool interaction during tool movement, which indicate that it is necessary to have information about size and shape of blank workpiece and to import that information in program in useful format. This paper present detail description of geometrical representation of blank which transforms blank from bitmap image to matrix record suitable for determination of its intersection with tool.

Module for machining parameters generation along tool path presents necessary base for development of methodology for choosing the most adequate tool path for machining of given contour considering minimum cutting force and dynamic stability..

Keywords: Tool path, matrix description of blank, peripheral milling,

\section{INTRODUCTION}

CAM programs can generate several different tool paths for same island or pocket peripheral machining. If optimal tool path is used, minimal cutting force, dynamic stability of process, minimum tool wear or some other criteria can be achieved.

Using CAM programs for creating tool path, technology designers faces some choices which determine the final shape of tool path. They use their experience, knowledge and intuition to choose some of offered options, so that generated tool path still largely depend of individual judgement. Figure 1. shows an example of different tool paths generated with the same CAM program for pocket machining.

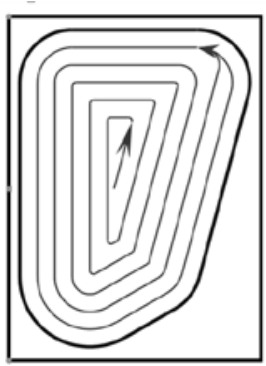

Figure1. Examples of typical tool paths generated by CAM program

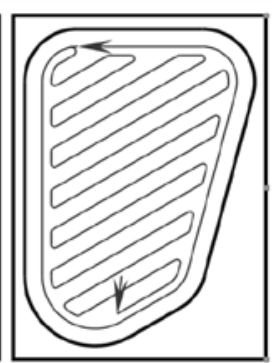

Tool path is conventionally generated based on the shape of contour (pocket or island), tool diameter and given step over so that the workpiece is completely machined.

Although the conventional tool path obtained from geometric information has been successful to make a desirable shape, it seldom considers physical process concerns like cutting forces and chatters [1].

Conventional tool-path strategies fail to adequately address concerns such as dynamic stability, acceleration *Author contact address: High technical mechanical school Trstenik, Radoja Krstića 19, 37240 Trstenik, Srbija, slobodan.ivanovic@vtmsts.edu.rs demands, and constantly varying tool engagement that limit production rates, increase tool wear, and reduce part quality [2]. That induce need for tool path optimization according to some physical criteria.

Tool path determines axial and radial depth of cut, engagement angle, feed and feed rate profile. Knowledge of the above machining elements along the tool path gives us insight into the adequacy of the generated tool path tools from different aspects.

\section{MODULE FOR MACHINING PARAMETERS GENERATION ALONG THE TOOL PATH}

Based on detailed analysis [3], for efficient controlling of cutting force along the path it is necessary to provide monitoring of cutting parameters along the tool path, primarily the radial depth of cut and cutting engagement angle, as well as monitoring of cutting force itself along the path. This parameters depend primarily of engagement zone. Accurate cutter/workpiece engagement (CWE) calculation is a key requirement for process modeling [4]. The simple examples will demonstrate the system developed to monitor parameters of milling process for such purposes.

First of all, NC code data generated by some of the CAM packages has to be imported the into a database and processed, that for each path segment the coordinates of start and end point, interpolation parameters, cutting conditions and the geometric characteristics of the tool are obtain, which is done according to the methodology given in [5].

Blank is imported in Matlab as a bitmap image representing view of blank from above, where each colour corresponds to an appropriate height of the blank. Figure 2 . shows the image of the blank and its appropriate matrix in 
Matlab, where you can see that a blank don't have to be prismatic, but may not have the horizontal holes.
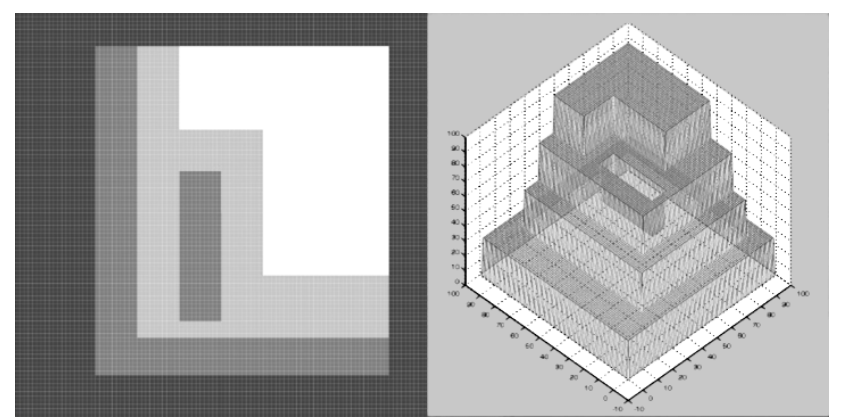

Figure 2. Bitmap image and blank matrix

The paper will deal extensively with this segment of module for machining parameters generation later.

Then, according to the processed data from the NC program, tool path and the cutter location relative to the workpiece are generated at appropriate spatial intervals along a path as shown in Figure 3.
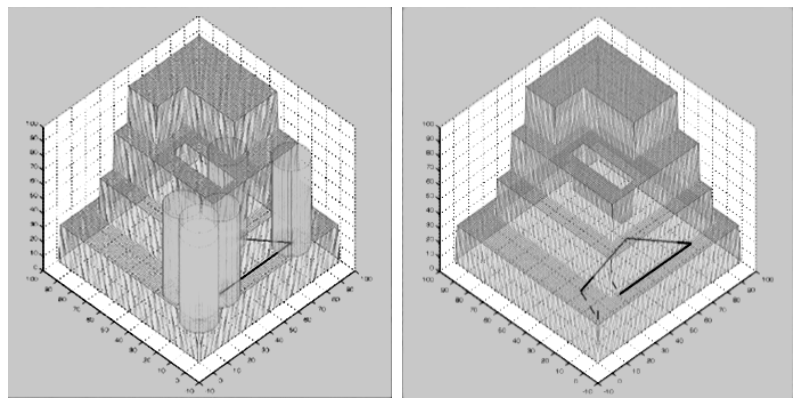

Figure 3. Cutter location relative to workpiece

Based on the cross-section of the cutting tool volume and the network of the blank, it is determined which section of the volume of the tool is in contact with the blank. In order to determine the angle of engagement and cutting depth in selected points of the tool path it is required to monitor section of the cutter half volume in the direction of the tool velocity in a bottom plane of tool, as well as at the axial depth of cut level (Figure 4).
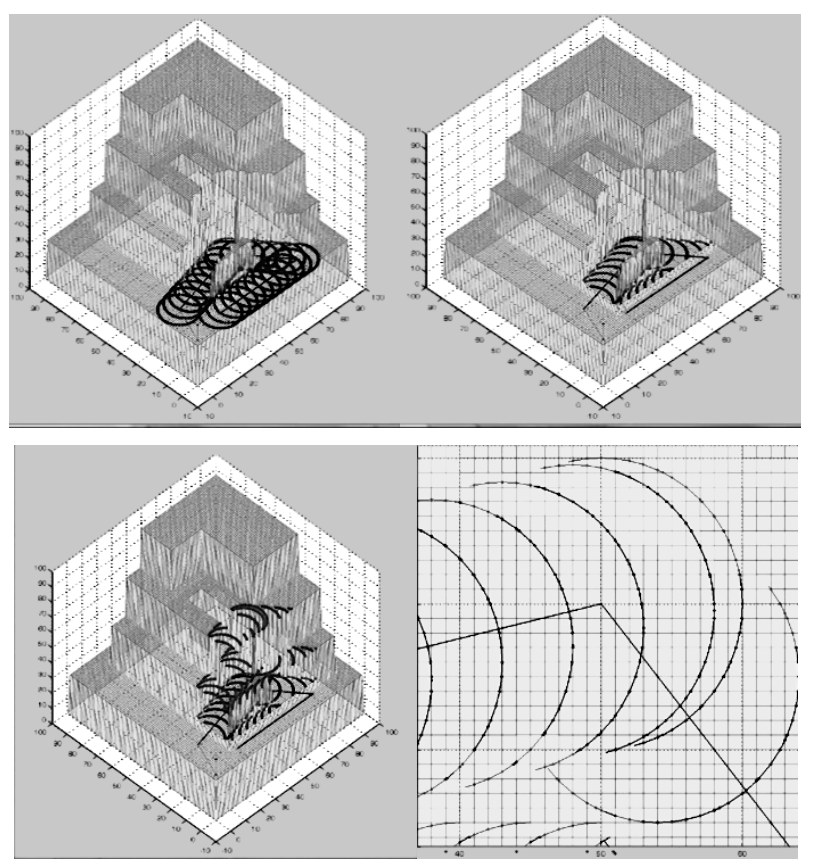

Figure 4. Cross-section of the cutting tool half volume and the network of the blank
Based on cross-section of cutting tool and blank network, values of radial depth of cut and cutting engagement angle are calculated at selected points of the tool path and diagrams of their variation along the tool path are generated (Figures 5, 6 and 7).

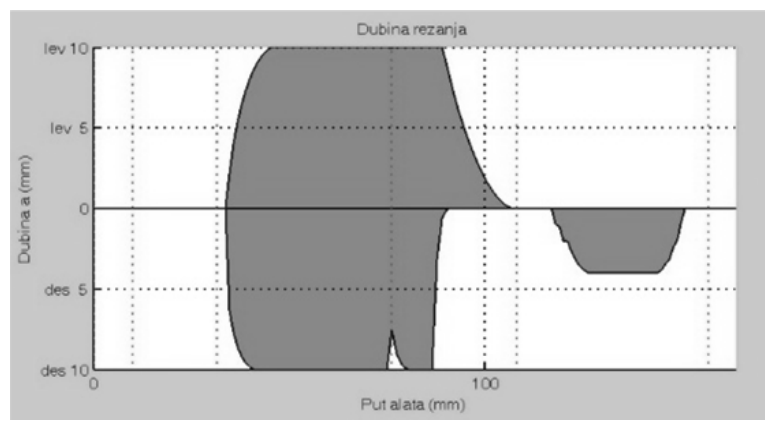

Figure 5. Radial depth of cut along the tool path

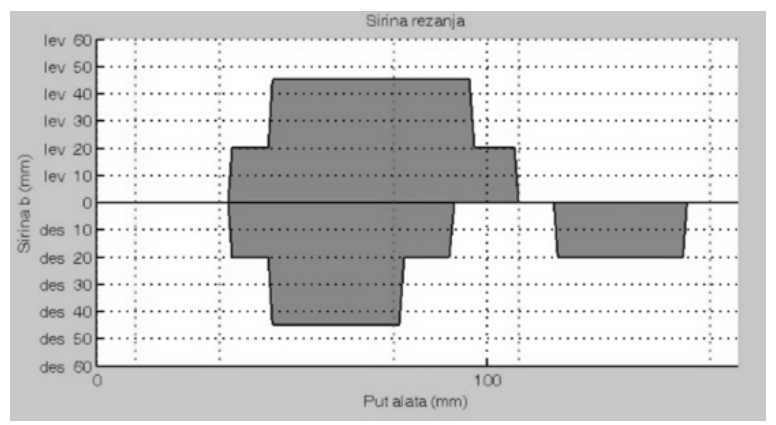

Figure 6. Axial depth of cut along the tool path

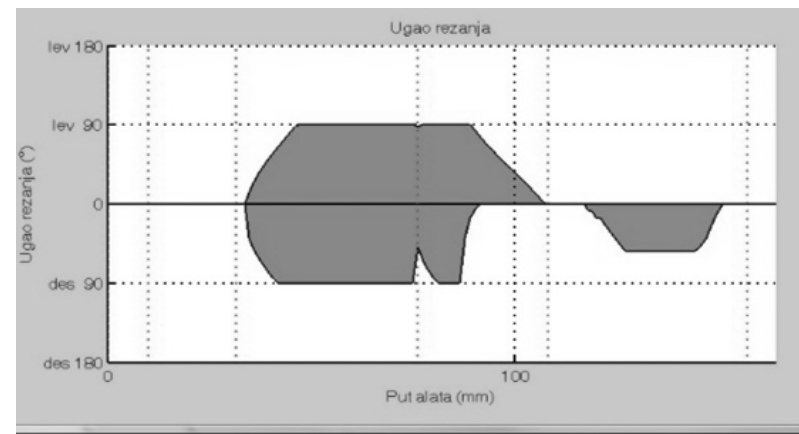

Figure 7. Cutting engagement angle along the tool path

One example of machining with flat end cutter is presented, although a developed program enables the use of tapered and spherical cutters.

On diagrams, values of the elements on the left and right side according to the direction of tool movement are shown separately, so that values that correspond to the up milling and ones that correspond to down milling can be separated.

Applying of the linear model of cutting forces described in [6] and engagement angle and axial depth of cut values obtained as shown above, it is possible to determine the value of the cutting force and its change along the tool path.

\section{GEOMETRICAL (MATRIX) BLANK DESCRIPTION}

Input information for geometrical description of blank (Figure 8b) is raster image of blank in $X Y$ plane (Figure 8a). Value of pixel colour determines height of blank surface. Pixel colour can take values of grey tones from 0 (black) to 255 (white). 


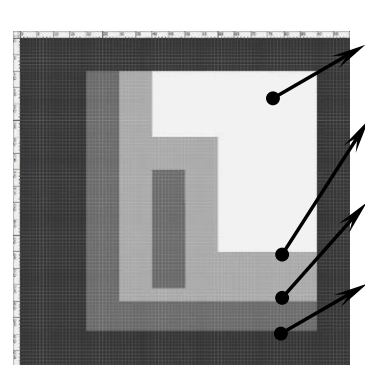

(a)

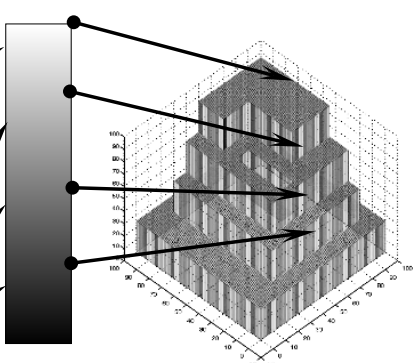

(b)
Slika 8. Blank transformation from bitmap image to matrix representation

Dimensions of raster blank base, coordinate and colour of each pixel are read from bitmap data (Figure 9) with difference in coordinate systems (Equation 1)

Matrix WC contains coloures of each pixel in coordinate plane $X Y$.

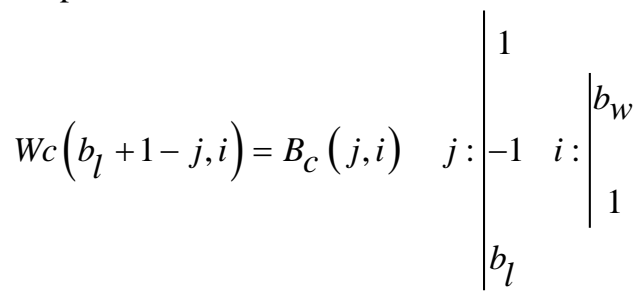

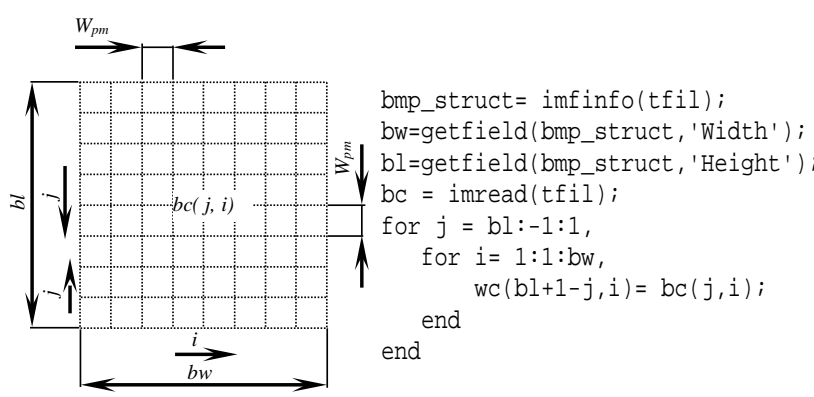

Slika 9. Bitmap data reading

Based on matrix $W c$, given maximum blank height $w_{z \text { max }}$ and given length of pixel in millimetres $w_{p m}$ matrices of point coordinates at blank net are calculated:

$$
\begin{gathered}
W x(j, i)=(i-1) \cdot w_{p m} \\
W y(j, i)=(j-1) \cdot w_{p m} \\
W z(j, i)= \\
=\left\{\begin{array}{c}
\text { NaN,Wc }(j, i)=0 \\
w_{\text {Zmax }} \cdot \frac{W_{c}(j, i)}{255}, 0<W c(j, i) \leq 255
\end{array} \mid \begin{array}{l}
b_{l} \\
1
\end{array}\right.
\end{gathered}
$$

If pixel colour is equal to 0 (black), in that point there is no surface (hole), and in contrary height of point at blank net $W z(j, i)$ is proportional to value of colour of coresponding pixel.

Number of division units at blank net in $x$ and $y$ direction is equal to number of pixels $b_{w}$ and $b_{l}$, and number of points on the blank net in same directions is $b_{w}+1$, and $b_{l}+1$, respectively. Because of that, it is necessary to enlarge matrices dimensions $W x, W y$ and $W z$ for 1 (Figure 10):

$$
\begin{array}{ll}
W x\left(j, b_{w}+1\right)=b_{w} \cdot w_{p m} & W x\left(b_{l}+1, i\right)=W x\left(b_{l}, i\right) \\
W y\left(j, b_{w}+1\right)=W y\left(j, b_{w}\right), & W y\left(b_{l}+1, i\right)=b_{l} \cdot w_{p m} \\
W z\left(j, b_{w}+1\right)=W z\left(j, b_{w}\right), & W z\left(b_{l}+1, i\right)=W z\left(b_{l}, i\right)
\end{array}
$$

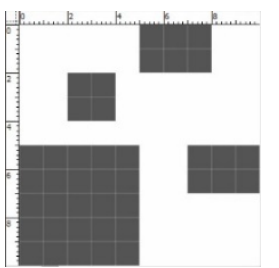

(a)

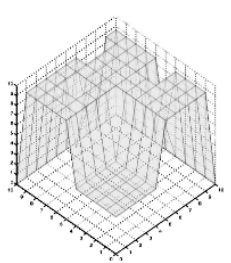

(b)

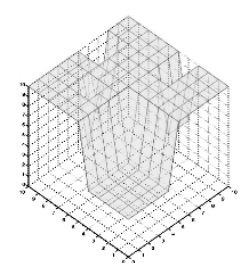

(c)
Figure 10. Matrices Wx, Wy and Wz dimension enlargement

Blank net $W$ is defined by matrices $W x$, Wy i $W z$, meaning:

$$
W=(W x, W y, W x)
$$

Accuracy of later machining elements calculations depends on dimension of matrices $W x, W y$ and $W z$, meaning it depends on matrix net resolution. In efort to get more accurate results, resolution of blank net $W$ is enlarged by adding net increment $w_{p m}$ to given pixel length $w_{p m}$ in millimetres (Figure 11). Then, $W x, W y$ and $W z$ matrix dimensions are:

$$
\begin{aligned}
& w_{j}=b_{l} \cdot w_{p m} \cdot w_{m p}+1 \\
& w_{i}=b_{w} \cdot w_{p m} \cdot w_{m p}+1
\end{aligned}
$$

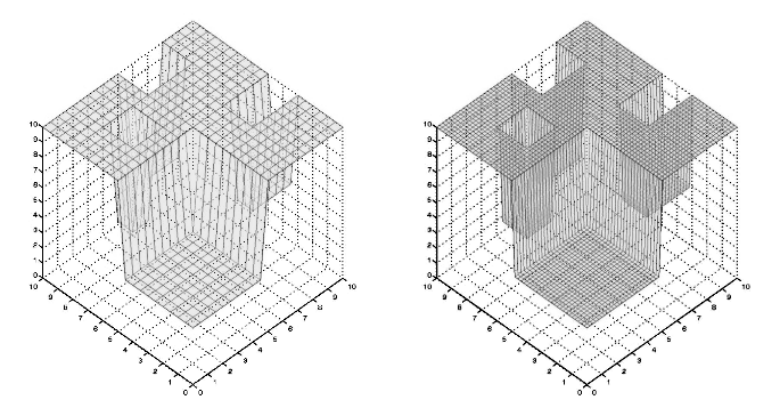

Figure 11. Different resolution of blank net $W$

Product $w_{p m} \cdot w_{m p}$ has to be integer and it represents number of points on pixel single unit. This product is equal to $j_{p p}$, or $i_{p p}$, respectively. Position of each point on net $(j$, i) is calculating through position of that point in corresponding pixel $\left(j_{p p}, i_{p p}\right)$ and pixel position on net $\left(j_{p}\right.$, $\left.i_{p}\right)$ :

$$
\begin{aligned}
& j_{p p}=\left\{\begin{array}{l}
w_{p m} \cdot w_{m p}, \quad j_{p}<b_{l} \\
w_{p m} \cdot w_{m p}+1, j_{p}=b_{l}
\end{array}\right. \\
& j=\left(j_{p}-1\right) \cdot w_{p m} \cdot w_{m p}+p_{j} \\
& i_{p p}=\left\{\begin{array}{l}
w_{p m} \cdot w_{m p}, \quad i_{p}<b_{w} \\
w_{p m} \cdot w_{m p}+1, i_{p}=b_{w}
\end{array}\right. \\
& i=\left(i_{p}-1\right) \cdot w_{p m} \cdot w_{m p}+p_{i}
\end{aligned}
$$

for: 


$$
\left.\underset{\substack{i_{p p} \\ i}}{j_{p p}} j_{p}^{b_{l}}\right|^{b_{j}}:\left.\right|_{1} ^{j_{p p}} i_{p}:\left.\right|_{1} ^{b_{w}} p_{i}:\left.\right|_{1} ^{i_{p p}}
$$

By determination of each point position on the net $(j$, i), instead of equations (2) and (3) matrices of point coordinates $W x, W y$ and $W z$ on blank workpiece net $W$ are calculated by:

$$
\begin{gathered}
W x(j, i)=\left[\left(i_{p}-1\right)+\frac{p_{i}-1}{w_{p m} \cdot w_{m p}}\right] \cdot w_{p m} \\
W y(j, i)=\left[\left(j_{p}-1\right)+\frac{p_{j}-1}{w_{p m} \cdot w_{m p}}\right] \cdot w_{p m} \\
W z(j, i)=\left\{\begin{array}{c}
N a N, \quad W c\left(j_{p}, i_{p}\right)=0 \\
w_{\text {zmax }} \cdot \frac{W c\left(j_{p}, i_{p}\right)}{255}, 0<W c\left(j_{p}, i_{p}\right) \leq 255
\end{array}\right.
\end{gathered}
$$

It is possible to move blank workpiece in adopted coordinate system $X Y Z$ by defining origin of coordinate system of the net $w_{x 0}, w_{y 0}, w_{z 0}$ (Figure 12), then:

$$
\begin{gathered}
W x(j, i)=w_{x 0}+\left[\left(i_{p}-1\right)+\frac{p_{i}-1}{w_{p m} \cdot w_{m p}}\right] \cdot w_{p m} \\
W y(j, i)=w_{y 0}+\left[\left(j_{p}-1\right)+\frac{p_{j}-1}{w_{p m} \cdot w_{m p}}\right] \cdot w_{p m} \\
W z(j, i)=N a N, \quad W c\left(j_{p}, i_{p}\right)=0 \\
=\left\{\begin{array}{c}
W c\left(j_{p}, i_{p}\right) \\
w_{z 0}+w_{z \max } \cdot \frac{W 5}{255}, 0<\left(j_{p}, i_{p}\right) \leq 255
\end{array}\right.
\end{gathered}
$$
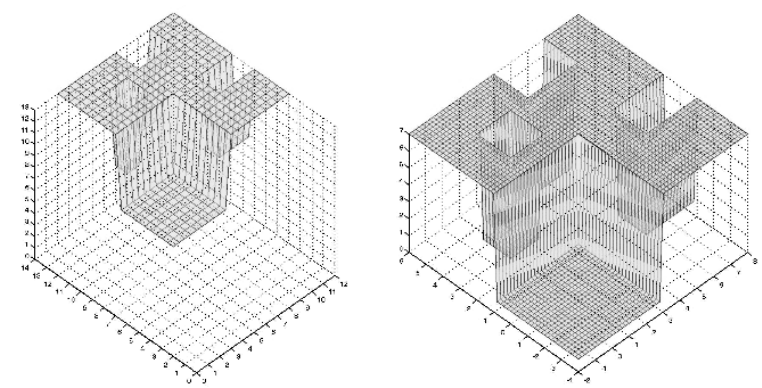

Figure12. Blank movement in coordinate system

Based on equation (9) it can be concluded that for one pair of coordinates $W x(j, i), W y(j, i)$ there is only one corresponding point on the net with height $W z(j, i)$. In that case it is impossible to define vertical edges of blank workpiece, holes, etc. For that reason, each position of the point on the net $j$ and $i$, respectively, is duplicated and positions $2 \cdot j-1$ and $2 \cdot j, 2 \cdot i-1$ and $2 \cdot i$, respectively, are obtained (Figure 13).
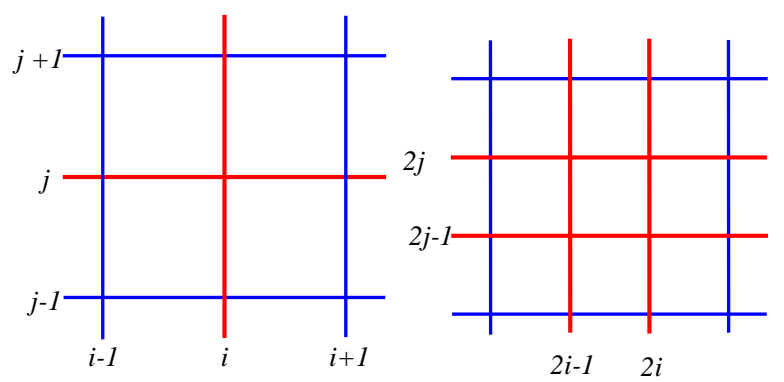

Figure 13. Positions $i$ and $j$ duplicating

Coordinates of obtained points on the net in matrices $W 2 x$ and $W 2 y$ are equal to coordinates $W x(j, i)$ and $W y(j, i)$ (Figure 14.), meaning:

$$
\begin{aligned}
& W 2 x(2 \cdot j-1,2 \cdot i-1)=W x(j, i), \\
& W 2 x(2 \cdot j, 2 \cdot i-1)=W x(j, i), \\
& W 2 x(2 \cdot j-1,2 \cdot i)=W x(j, i) \\
& W 2 x(2 \cdot j, 2 \cdot i)=W x(j, i) \\
& W 2 y(2 \cdot j-1,2 \cdot i-1)=W y(j, i), \\
& W 2 y(2 \cdot j, 2 \cdot i-1)=W y(j, i) \\
& W 2 y(2 \cdot j-1,2 \cdot i)=W y(j, i) \\
& W 2 y(2 \cdot j, 2 \cdot i)=W y(j, i)
\end{aligned}
$$

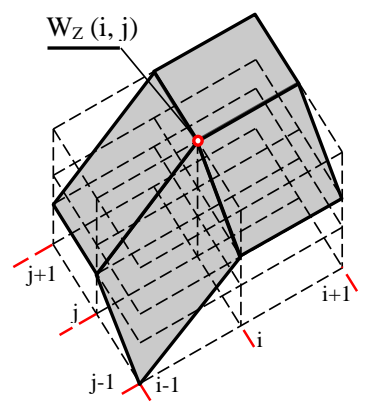

(a)

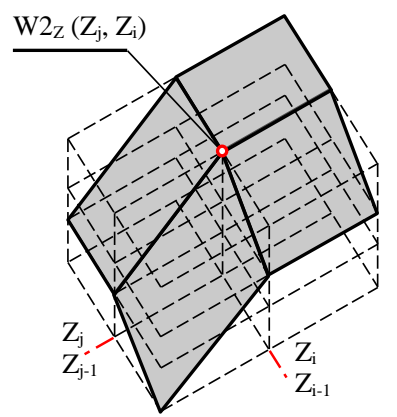

(b)
Figure 14. Points coordinates on the net in matrices $W 2 x$ and $W 2 y$ and coordinates $W x(j, i)$ and $W y(j, i)$

Coordinate $W 2 z(2 \cdot j, 2 \cdot i)$ is equal to coordinate $W z(j, i)$ (Figure 14.), while the coordinates of the other points on the net in matrix $W 2 z$, are equal to coordinates of coresponding points in matrix $\mathrm{Wz}$ (Figure15.):

$$
\begin{aligned}
W 2 z(2 \cdot j-1,2 \cdot i-1) & = \begin{cases}W z(j-1, i-1), j>1 \wedge i>1 \\
0, & j=1 \vee i=1\end{cases} \\
W 2 z(2 \cdot j, 2 \cdot i-1) & = \begin{cases}W z(j, i-1), & j<w_{j} \wedge i>1 \\
0, & j=w_{j} \vee i=1\end{cases} \\
W 2 z(2 \cdot j-1,2 \cdot i) & = \begin{cases}W z(j-1, i), & j>1 \wedge i<w_{i} \\
0, & j=1 \vee i=w_{i}\end{cases} \\
W 2 z(2 \cdot j, 2 \cdot i) & = \begin{cases}W z(j, i), j<w_{j} \wedge i<w_{i} \\
0, & j=w_{j} \vee i=w_{i}\end{cases}
\end{aligned}
$$

for:

$$
\begin{aligned}
& W 2 z(2 \cdot j-1,2 \cdot i-1) \\
& W 2 z(2 \cdot j, 2 \cdot i-1) \\
& W 2 z(2 \cdot j-1,2 \cdot i) \\
& W 2 z(2 \cdot j, 2 \cdot i)
\end{aligned} \quad j:\left.\right|_{1} ^{w_{j}} \quad i:\left.\right|_{1} ^{w_{i}}
$$




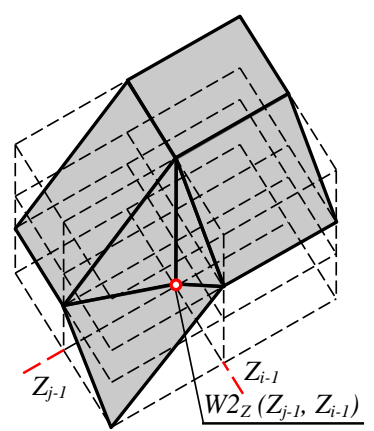

(a)

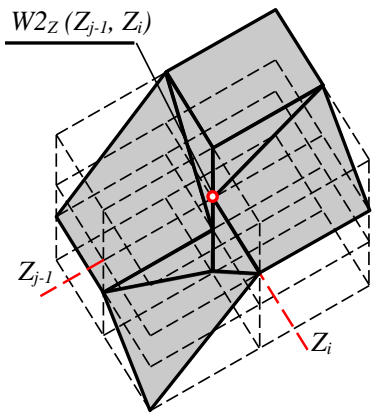

(c)

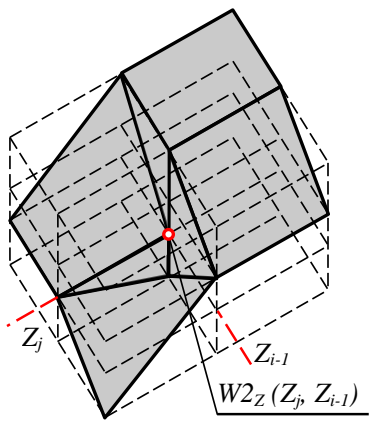

(b)

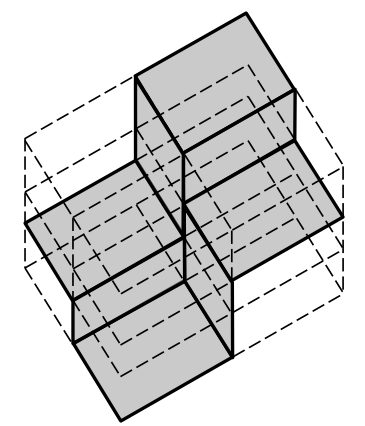

(d)
Figure 15. Coordinates of coresponding points in matrix $\mathrm{Wz}$
Blank net $W 2$ is defined by matrices $W 2 x, W 2 y$ and W2z:

$$
W 2=(W 2 x, W 2 y, W 2 x)
$$

Dimensions of points coordinates matrices $W 2 x$, $W 2 y$ and $W 2 z$ at blank net $W 2$ are equal:

$$
\begin{aligned}
& w 2_{j}=2 \cdot w_{j} \\
& w 2_{i}=2 \cdot w_{i}
\end{aligned}
$$

By transforming blank net $W$ to net $W 2$ from shape at figure 14.a the shape at figure 15.d is get. In that way, the blank nets presented at figures 10.c and 11 are transformed to nets shown at figure 16 .

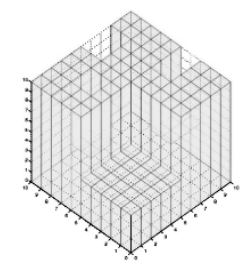

(a)

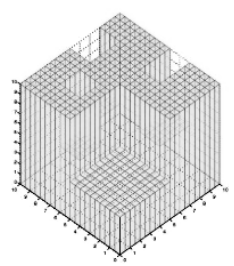

(б)

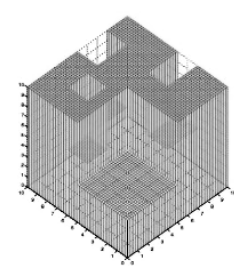

(B)
Figure 16. Blank nets

Matrices of points coordinates $W x, W y, W z, W 2 x$, $W 2 y$ and $W 2 z$ are written in ASCII files (Figure 17- example for presented at figure 16.a), as well as other information about blank workpiece.

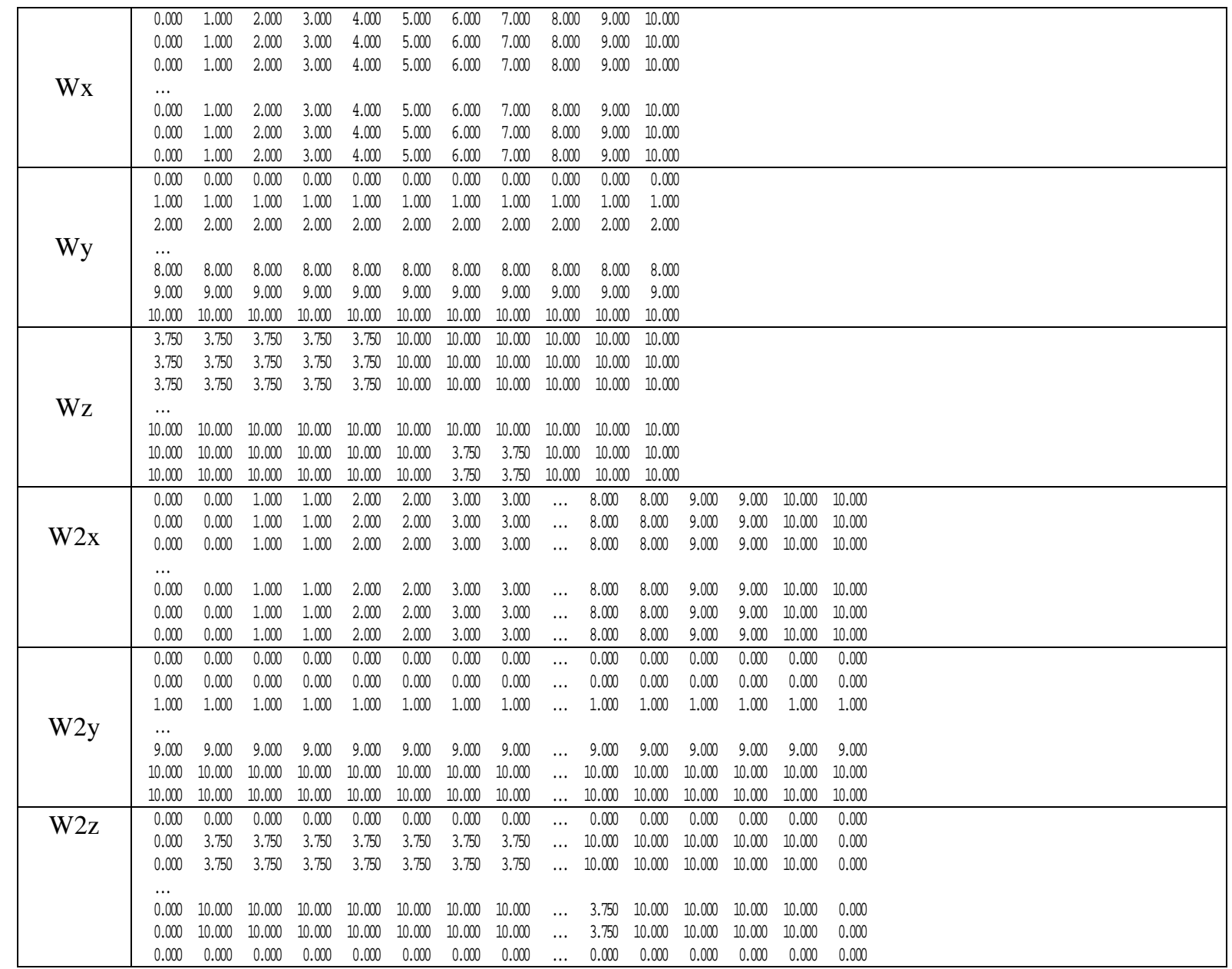

Figure 17. Example of ASCII data for net presented at figure 16.a 
Described methodology allows transformation of raster blank base in $X Y$ plane stored in bitmap data (Figure 8.a) to blank workpiece net $W$, and $W 2$, respectievly (Figure 8.b).

\section{CONCLUSION}

The program developed to simulate the processing parameters allows us to track changes in depth and width of the cut and engagement angle along the tool path. On the basis of these variations, cutting force during the milling process can be monitored. Based on the analysis of the cutting force diagram for the different tool paths, in the future work a methodology for assessing the tool path and selection of the most appropriate path from the point of minimum cutting force variations will be develop. The analysis can also include other criteria such as tool wear, the total processing time and process stability. Also, it is required to include the impact of feed rate variability in points of changing tool velocity direction. This would allow to comprehend all aspects of the cutting process and enable selection of the optimal tool path.

Methodology of optimal tool path determination is presented at figure 18. In this paper some of steps are described. Program which support this methodology is completely developed, and very complex. Here is presented detailed explanation of the step "blank matrix description", which belong to preparatory stage. Preparatory and calculation stage are realized in Matlab and together they make module for generation of machining parameters along the tool path.

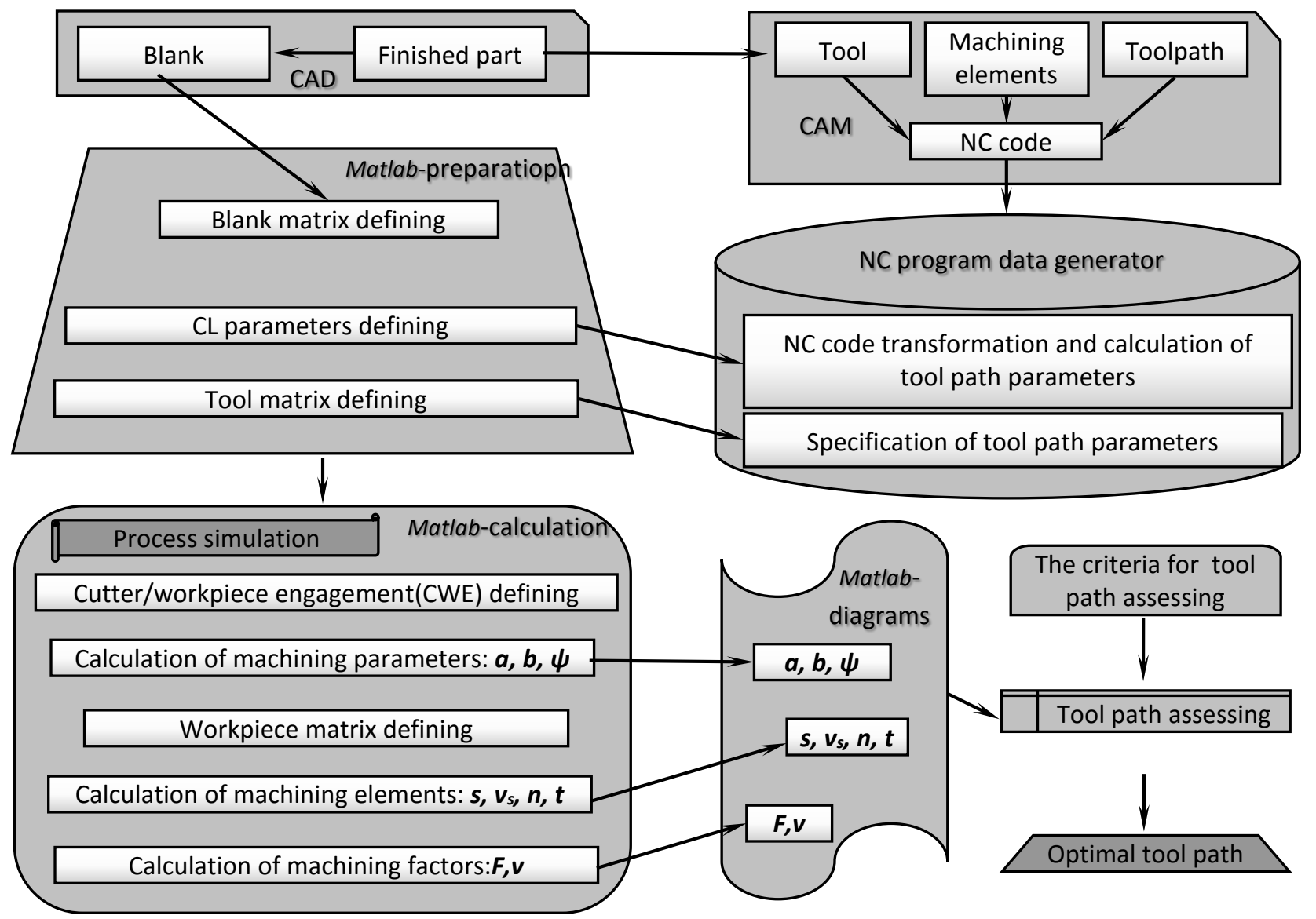

Slika 18. Struktura procesa određivanja optimalne putanje alata

\section{LITERATURE}

[1] H.-C. Kim, “Tool path modification for optimized pocket milling,” Int. J. Prod. Res., vol. 45, no. 24, pp. 5715-5729, Dec. 2007.

[2] H. Wang, P. Jang, and J. A. Stori, “A MetricBased Approach to Two-Dimensional (2D) Tool-Path Optimization for High-Speed Machining," J. Manuf. Sci. Eng., vol. 127, no. 1, p. 33, Feb. 2005.

[3] A. Petrović, S. Ivanović, and L. Lukić, "NC Tool Path and Its Influence on Cutting Force in Peripheral Milling Analyses,” IMK -14-- Res. Dev. Heavy Mach., vol. 21, pp. 41-46, 2015.

[4] D. Yip-Hoi and X. Huang, "Cutter/Workpiece Engagement Feature Extraction from Solid Models for
End Milling,” J. Manuf. Sci. Eng., vol. 128, no. 1, p. 249, Feb. 2006.

[5] A. Petrović, S. Ivanović, and L. Lukić, "GENERATING PARAMETERS FROM THE NC CODE NEEDED FOR DEFINING THE OPTIMAL TOOL PATH,” in MMA 2015 - FLEXIBLE TEHNOLOGIES, 2015.

[6] A. Petrović, L. Lukić, and M. Pljakić, "Machining Parameters Influence on Cutting Force Used for Tool Path Optimizafzion in End Milling," in Proceedings of the 8th International Conference Heavy Machinery HM 2014, 2014, p. B 19-25. 